\title{
COLLABORATIVE OLAP WITH TAG CLOUDS Web 2.0 OLAP Formalism and Experimental Evaluation
}

\author{
Kamel Aouiche, Daniel Lemire and Robert Godin \\ Université du Québec à Montréal, 100 Sherbrooke West, Montreal, Canada \\ kamel.aouiche@gmail.com,lemire@acm.org,godin.robert@uqam.ca
}

Keywords: $\quad$ OLAP, Data Warehouse, Business Intelligence, Tag Cloud, Social Web

\begin{abstract}
Increasingly, business projects are ephemeral. New Business Intelligence tools must support ad-lib data sources and quick perusal. Meanwhile, tag clouds are a popular community-driven visualization technique. Hence, we investigate tag-cloud views with support for OLAP operations such as roll-ups, slices, dices, clustering, and drill-downs. As a case study, we implemented an application where users can upload data and immediately navigate through its ad hoc dimensions. To support social networking, views can be easily shared and embedded in other Web sites. Algorithmically, our tag-cloud views are approximate range top-k queries over spontaneous data cubes. We present experimental evidence that iceberg cuboids provide adequate online approximations. We benchmark several browser-oblivious tag-cloud layout optimizations.
\end{abstract}

\section{INTRODUCTION}

The Web 2.0, or Social Web, is about making available social software applications on the Web in an unrestricted manner. Enabling a wide range of distributed individuals to collaborate on data analysis tasks may lead to significant productivity gains ( $\mathrm{Heer}$ et al., 2007; Wattenberg and Kriss, 2006). Several companies, like SocialText and IBM, are offering Web 2.0 solutions dedicated to enterprise needs. The data visualization Web sites Many Eyes (IBM, 2007) and Swivel (Swivel, Inc, 2007) have become part of the Web 2.0 landscape: over 1 million data sets were uploaded to Swivel in less than 3 months (Butler, 2007).

These Web 2.0 data visualization sites use traditional pie charts and histograms, but also tag clouds. Tag clouds are a form of histogram which can represent the amplitude of over a hundred items by varying the font size. The use of hyperlinks makes tag clouds naturally interactive. Tag clouds are used by many Web 2.0 sites such as Flickr, del.icio.us and Technorati. Increasingly, e-Commerce sites such as Amazon or O'Reilly Media, are using tag clouds to help their users navigate through aggregated data.

Meanwhile, OLAP (On-Line Analytical Process- ing) (Codd, 1993) is a dominant paradigm in Business Intelligence (BI). OLAP allows domain experts to navigate through aggregated data in a multidimensional data model. Standard operations include drilldown, roll-up, dice, and slice. The data cube (Gray et al., 1996) model provides well-defined semantics and performance optimization strategies. However, OLAP requires much effort from database administrators even after the data has been cleaned, tuned and loaded: schemas must be designed in collaboration with users having fast changing needs and requirements (Body et al., 2002, Morzy and Wrembel, 2004). Vendors such as Spotfire, Business Objects and QlikTech have reacted by proposing a new class of tools allowing end-user to customize their applications and to limit the need for centralized schema crafting (Havenstein, 2003).

OLAP itself has never been formally defined though rules have been proposed to recognize an OLAP application (Codd, 1993). In a similar manner, we propose rules to recognize Web 2.0 OLAP applications (see also Table 1):

1. Data and schemas are provided autonomously by users.

2. It is available as a Web application. 
3. It supports complete online interaction over aggregated multidimensional data.

4. Users are encouraged to collaborate.

Tag clouds are well suited for Web 2.0 OLAP. They are flexible: a tag cloud can represent a dozen or hundred different amplitudes. And they are accessible: the only requirement is a browser that can display different font sizes.

We describe a tag-cloud formalism, as an instance of Web 2.0 OLAP. Since we implemented a prototype, technical issues will be discussed regarding application design. In particular, we used iceberg cubes (Carey and Kossmann, 1997) to generate tag clouds online when the data and schema are provided extemporaneously. Because tag clouds are meant to convey a general impression, presenting approximate measures and clustering is sufficient: we propose specific metrics to measure the quality of tag-cloud approximations. We conclude the paper with experimental results on real and synthetic data sets.

Table 1: Conventional OLAP versus Web 2.0 OLAP

\begin{tabular}{|l|l|}
\hline Conventional OLAP & Web 2.0 OLAP \\
\hline recurring needs & ephemeral projects \\
predefined schemas & spontaneous schemas \\
centralized design & user initiative \\
histograms & tag clouds \\
plots and reports & iframes, wikis, blogs \\
access control & social networking \\
\hline
\end{tabular}

\section{RELATED WORK}

There are decentralized models (Taylor and Ives, 2006) and systems (Green et al., 2007) to support collaborative data sharing without a single schema.

According to $\mathrm{Wu}$ et al., it is difficult to navigate an OLAP schema without help; they have proposed a keyword-driven OLAP model (Wu et al., 2007). There are several OLAP visualization techniques including the Cube Presentation Model (CPM) (Maniatis et al., 2005), Multiple Correspondence Analysis (MCA) (Ben Messaoud et al., 2006) and other interactive systems (Techapichetvanich and Datta, 2005).

Tag clouds have been popularized by the Web site Flickr launched in 2004. Several optimization opportunities exist: similar tags can be clustered together (Kaser and Lemire, 2007), tags can be pruned automatically (Hassan-Montero and Herrero-Solana, 2006) or by user intervention (Millen et al., 2006), tags can be indexed (Millen et al., 2006), and so on. Tag clouds can be adapted to spatio-temporal data (Russell, 2006; Jaffe et al., 2006).

\section{OLAP FORMALISM}

\subsection{Conventional OLAP Formalism}

Most OLAP engines rely on a data cube (Gray et al., 1996). A data cube $C$ contains a non empty set of $d$ dimensions $\mathcal{D}=\left\{D_{i}\right\}_{1 \leq i \leq d}$ and a non empty set of measures $\mathcal{M}$. Data cubes are usually derived from a fact table (see Table 2) where each dimension and measure is a column and all rows (or facts) have disjoint dimension tuples. Figure 1(a) gives tridimensional representation of the data cube.

Table 2: Fact table example

\begin{tabular}{|llll|cc|}
\hline \multicolumn{5}{c|}{ Dimensions } & \multicolumn{2}{c|}{ Measures } \\
\hline location & time & salesman & product & cost & profit \\
\hline Montreal & March & John & shoe & $100 \$$ & $10 \$$ \\
Montreal & December & Smith & shoe & $150 \$$ & $30 \$$ \\
Quebec & December & Smith & dress & $175 \$$ & $45 \$$ \\
Ontario & April & Kate & dress & $90 \$$ & $10 \$$ \\
Paris & March & John & shoe & $100 \$$ & $20 \$$ \\
Paris & March & Marc & table & $120 \$$ & $10 \$$ \\
Paris & June & Martin & shoe & $120 \$$ & $5 \$$ \\
Lyon & April & Claude & dress & $90 \$$ & $10 \$$ \\
New York & October & Joe & chair & $100 \$$ & $10 \$$ \\
New York & May & Joe & chair & $90 \$$ & $10 \$$ \\
Detroit & April & Jim & dress & $90 \$$ & $10 \$$ \\
\hline
\end{tabular}

Measures can be aggregated using several operators such as AVERAGE, MAX, MIN, SUM, and COUNT. All of these measures and dimensions are typically prespecified in a database schema. Database administrators preaggregate views to accelerate queries.

The data cube supports the following operations:

- A slice specifies that you are only interested in some attribute values of a given dimension. For example, one may want to focus on one specific product (see Figure $1(\mathrm{~g})$. Similarly, a dice selects ranges of attribute values (see Figure 1(e)].

- A roll-up aggregates the measures on coarser attribute values. For example, from the sales given for every store, a user may want to see the sales aggregated per country (see Figure $1(\mathrm{c})$ ). A drilldown is the reverse operation: from the sales per country, one may want to explore the sales per store in one country.

The various specific multidimensional views in Figure 1 are called cuboids.

\subsection{Tag-Cloud OLAP Formalism}

A Web 2.0 OLAP application should be supported by a flexible formalism that can adapt a wide range of 


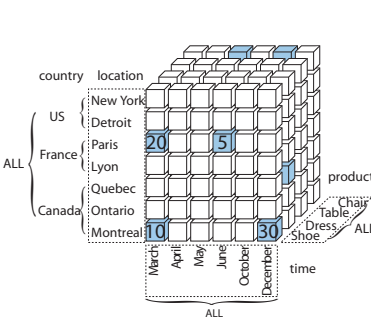

(a) OLAP data cube

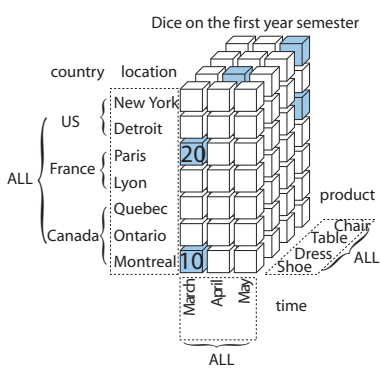

(e) OLAP dice

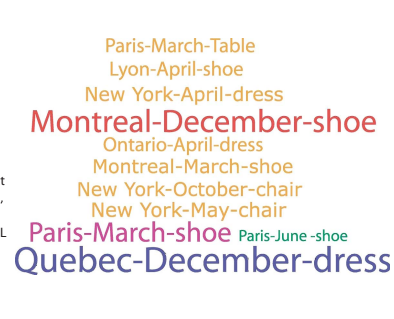

(b) Tag-cloud data cube

\section{New York-May-chair Lyon-April-shoe}

New York-April-dress Ontario-April-dress

Montreal-March-shoe

Paris-March-Table

Paris-March-shoe

(f) Tag-cloud dice

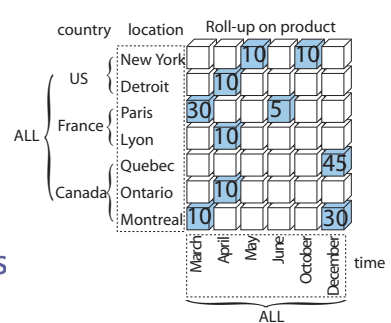

(c) OLAP roll-up

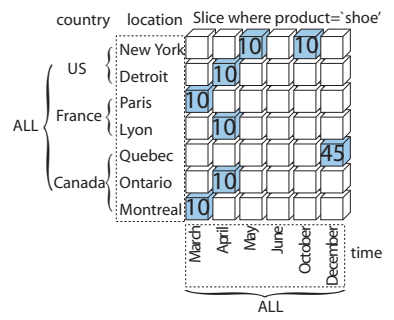

(g) OLAP slice

\section{Lyon-April \\ Montreal-December Montreal-March}

New York-April New York-May

New York-October Ontario-April

Paris-March Paris-June Quebec-December

(d) Tag-cloud roll-up

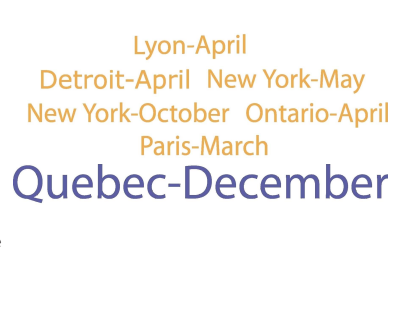

(h) Tag-cloud slice

Figure 1: Conventional OLAP operations vs. tag-cloud OLAP operations

data loaded by users. Processing time must be reasonable and batch processing should be avoided.

Unlike in conventional data cubes, we do not expect that most dimensions have explicit hierarchies when they are loaded: instead, users can specify how the data is laid out (see Section 5). As a related issue, the dimensions are not orthogonal in general: there might be a "City" dimension as a well as "Climate Zone" dimension. It is up to the user to organize the cities per climate zone or per country.

Definition 1 (Tag) A tag is a term or phrase describing an object with corresponding non-negative weights determining its relative importance. Hence, a tag is made of a triplet (term, object, weight).

As an example, a picture may have been attributed the tags "dog" (12 times) and "cat" (20 times). In a Business Intelligence context, a tag may describe the current state of a business. For example, the tags "USA" $(16,000 \$)$ and "Canada" $(8,000 \$)$ describe the sales of a given product by a given salesman.

We can aggregate several attribute values, such as "Canada" and "March," into a single term, such as "Canada-March." A tag composed of $k$ attribute values is called a $k$-tag. Figure $1(\mathrm{~b})$ shows a tag cloud representation of Table 2 using 3-tags.

Each tag $T$ is represented visually using a font size, font color, background color, area or motif, depending on its measure values.

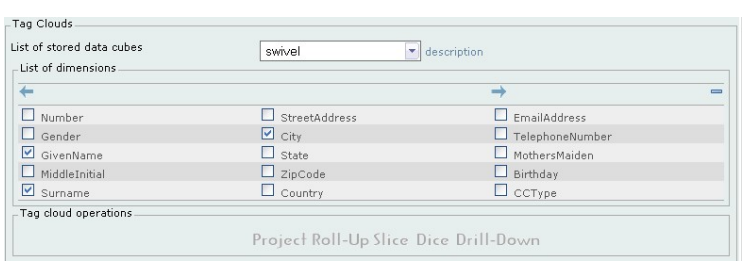

Figure 2: User-driven schema design

\subsection{Tag-Cloud Operations}

In our system, users can upload data, select a data set, and define a schema by choosing dimensions (see Figure 2). Then, users can apply various operations on the data using a menu bar. On the one hand, OLAP operations such as slice, dice, roll-up and drill-down generate new tag clouds and new cuboids from existing cuboids. Figures 1(d), 1(f) and 1(h), show the results of a roll-up, a dice, and a slice as tag clouds. On the other hand, we can apply some operations on an existing tag cloud: sort by either the weights or the terms of tags, remove some tags, remove lesser weighted tags, and so on. We estimate that a tag cloud should not have more than 150 tags.

Tag-cloud layout has measurable benefits when trying to convey a general impression (Rivadeneira et al., 2007). Hence, we wish to optimize the visual 


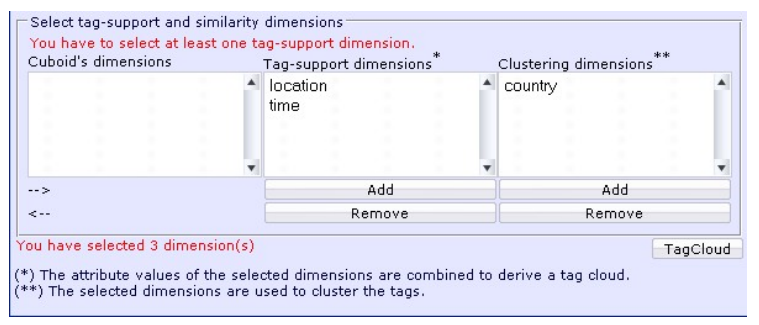

Figure 3: Choosing similarity dimensions

arrangement of tags. Chen et al. propose the computation of similarity measures between cuboids to help users explore data (Chen et al., 2000): we apply this idea to define similarities between tags. First of all, users are asked to provide one or several dimensions they want to use to cluster the tags. Choosing the "Country" dimension would mean that the user wants the tags rearranged by countries so that "Montreal-April" and "Toronto-March" are nearby (see Figure 3). The clustering dimensions selected by the user together with the tag-cloud dimensions form a cuboid: in our example, we have the dimensions "Country," "City," and "Time." Since a tag contains a set of attribute values, it has a corresponding $s u b$ cuboid defined by slicing the cuboid.

Several similarity measures can be applied between subcuboids: Jaccard, Euclidean distance, cosine similarity, Tanimoto similarity, Pearson correlation, Hamming distance, and so on. Which similarity measure is best depends on the application at hand, so advanced users should be given a choice. Commonly, similarity measures take up values in the interval $[-1,1]$. Similarity measures are expected to be reflexive $(f(a, a)=1)$, symmetric $(f(a, b)=f(b, a))$ and transitive: if $a$ is similar to $b$, and $b$ is similar to $\mathrm{C}$, then a is also similar to $\mathrm{C}$.

Recall that given two vectors $v$ and $w$, the cosine similarity measure is defined as $\cos (v, w)=$ $\sum_{i} v_{i} w_{i} / \sqrt{\sum_{i} v_{i}^{2} \sum_{i} w_{i}^{2}}=v /|v| \cdot w /|w|$. The Tanimoto similarity is given by $\sum_{i} v_{i} w_{i} /\left(\sum_{i} v_{i}^{2}+\sum_{i} w_{i}^{2}-\right.$ $\left.\sum_{i} v_{i} w_{i}\right)$; it becomes the Jaccard similarity when the vectors have binary values. Both of these measures are reflexive, symmetric and transitive. Specifically, the cosine similarity is transitive by this inequality: $\cos (v, z) \geq \cos (w, z)-\sqrt{1-\cos (v, w)^{2}}$. To generalize the formulas from vectors to cuboids, it suffices to replace the single summation by one summation per dimension. Figure 4 shows an example of tag-cloud reordering to cluster similar tags. In this example, the "City-Product" tags were compared according to the "Country" dimension. The result is that the tags are clustered by countries.

Without similarity
\begin{tabular}{|l|l|}
\hline Detroit-dress Quebec-dress Paris-table Ontario-dress \\
Paris-shoe Montreal-shoe Lyon-dress New York-chair
\end{tabular}
With similarity
$\begin{aligned} & \text { Detroit-dress New York-chair Quebec-dress } \\
& \text { Ontario-dressMontreal-shoeparis-tableParis-shoelyon-dress }\end{aligned}$

Figure 4: Tag-cloud reordering based on similarity

\section{FAST COMPUTATION}

Because only a moderate number of tags can be displayed, the computation of tag clouds is a form of top- $k$ query: given any user-specified range of cells, we seek the top- $k$ cells having the largest measures. There is a little hope of answering such queries in near constant-time with respect to the number of facts without an index or a buffer. Indeed, finding all and only the elements with frequency exceeding a given frequency threshold (Cormode and Muthukrishnan, 2005) or merely finding the most frequent element (Alon et al., 1996) requires $\Omega(m)$ bits where $m$ is the number of distinct items.

Various efficient techniques have been proposed for the related range MAX problem (Chazelle, 1988 Poon, 2003), but they do not necessarily generalize. Instead, for the range top- $k$ problem, we can partition sparse data cubes into customized data structures to speed up queries by an order of magnitude (Luo et al., 2001; Loh et al., 2002a, Loh et al., 2002b). We can also answer range top- $k$ queries using RDtrees (Chung et al., 2007) or R-trees (Seokjin et al., 2005). In tag clouds, precision is not required and accuracy is less important; only the most significant tags are typically needed. Further, if all tags have similar weights, then any subset of tag may form an acceptable tag cloud.

A strategy to speed up top- $k$ queries is to transform them into comparatively easier iceberg queries (Carey and Kossmann, 1997). For example, in computing the top-10 $(k=10)$ best vendors, one could start by finding all vendors with a rating above $4 / 5$. If there are at least 10 such vendors, then sorting this smaller list is enough. If not, one can restart the query, seeking vendors with a rating above $3 / 5$. Given a histogram or selectivity estimates, we can reduce the number of expected iceberg queries (Donjerkovic and Ramakrishnan, 1999). Unfortunately, this approach is not necessarily applicable to multidimensional data since even computing iceberg aggre- 


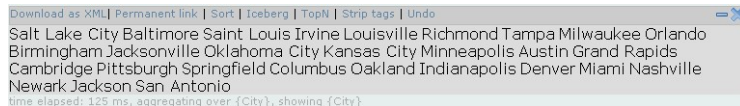

Figure 5: Example of non informative tag cloud

gates once for each query may be prohibitive. However, iceberg cuboids can still be put to good use. That is, one materializes the iceberg of a cuboid, small enough to fit in main memory, from which the tag clouds are computed. Intuitively, a cuboid representing the largest measures is likely to provide reasonable tag clouds. Users mostly notice tags with large font sizes (Rivadeneira et al., 2007). A good approximation captures the tags having significantly larger weights. To determine whether a tag cloud has such significant tags, we can compute the entropy.

Definition 2 (Entropy of a tag cloud) Let $T \in \mathcal{T}$ be a tag from a tag cloud $\mathcal{T}$, then entropy $(\mathcal{T})=$ $-\sum_{T \in \mathcal{T}} p(T) \log (p(T))$ where $p(T)=\frac{\text { weight }(T)}{\sum_{x \in \mathcal{T}} \text { weight }(x)}$.

The entropy quantifies the disparity of weights between tags. The lower the entropy, the more interesting the corresponding tag cloud is. Indeed, tag clouds with uniform tag weights have maximal entropy and are visually not very informative (see Figure 5).

We can measure the quality of a low-entropy tag cloud by measuring false positives and negatives: false positive happens when a tag has been falsely added to a tag cloud whereas a false negative occurs when a tag is missing. These measures of error assume that we limit the number of tags to a moderately small number. We use the following quality indexes; index values are in $[0,1]$ and a value of 0 is ideal; they are not applicable to high-entropy tag clouds.

Definition 3 Given approximate and exact tag clouds $A$ and $E$, the false-positive and false-negative indexes are $\frac{\max _{t \in A, t \notin E} \text { weight }(t)}{\max _{t \in A} \text { weight }(t)}$ and $\frac{\max _{t \in E, t \notin A} \text { weight }(t)}{\max _{t \in E} \text { weight }(t)}$.

\section{TAG-CLOUD DRAWING}

While we can ensure some level of deviceindependent displays on the Web, by using images or plugins, text display in HTML may vary substantially from browser to another. There is no common set of font browsers are required to support, and Web standards do not dictate line-breaking algorithms or other typographical issues. It is not practical to simulate the browser on a server. Meanwhile, if we wish to remain accessible and to abide by open standards, producing HTML and ECMAScript is the favorite option.
Given tag-cloud data, the tag-cloud drawing problem is to optimally display the tags, generally using HTML, so that some desirable properties are met, including the following: (1) the screen space usage is minimized; (2) when applicable, similar tags are clustered together. Typically, the width of the tag cloud is fixed, but its height can vary.

For practical reasons, we do not wish for the server to send all of the data to the browser, including a possibly large number of similarity measures between tags. Hence, some of the tag-cloud drawing computations must be server-bound. There are two possible architectures. The first scenario is a browseraware approach (Kaser and Lemire, 2007): given the tag-cloud data provided by the server, the browser sends back to the server some display-specific data, such as the box dimensions of various tags using different font sizes. The server then sends back an optimized tag cloud. The second approach is browseroblivious: the server optimizes the display of the tag cloud without any knowledge of the browser by passing simple display hints. The browser can then execute a final and inexpensive display optimization. While browser-oblivious optimization is necessarily limited, it has reduced latency and it is easily cacheable.

Browser-oblivious optimization can take many forms. For example, we could send classes of tags and instruct the browser to display them on separate lines (Hassan-Montero and Herrero-Solana, 2006). In our system, tags are sent to the browser as an ordered list, using the convention that successive tags are similar and should appear nearby. Given a similarity measure $w$ between tags, we want to minimize $\sum_{p, q} w(p, q) d(p, q)$ where $d(p, q)$ is a distance function between the two tags in the list and the sum is over all tags. Ideally, $d(p, q)$ should be the physical distance between the tags as they appear in the browser; we model this distance with the index distance: if tag $a$ appears at index $i$ in the list and $\operatorname{tag} b$ appears at index $j$, their distance is the integer $|i-j|$. This optimization problem is an instance of the NP-complete MINIMUM LINEAR ARRANGEMENT (MLA) problem: an optimal linear arrangement of a graph $G=(V, E)$, is a map $f$ from $V$ onto $\{1,2, \ldots, N\}$ minimizing $\sum_{u, v \in V}|f(u)-f(v)|$.

Proposition 1 The browser-oblivious tag-cloud optimization problem is NP-Complete.

There is an $\mathrm{O}(\sqrt{\log n} \log \log n)$-approximation for the MLA problem (Feige and Lee, 2007) in some instances. However, for our generic purposes, the greedy NEAREST NEIGHBOR (NN) algorithm might suffice: insert any tag in an empty list, then repeatedly append a tag most similar to the latest tag in 


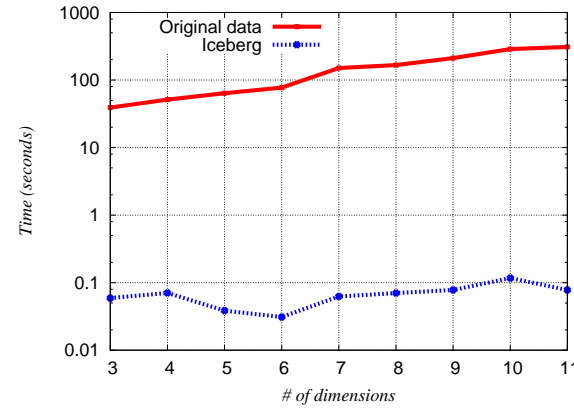

Figure 6: Computing tag clouds from original data vs. icebergs: iceberg limit value set at 150 and tag-cloud size is 9 (US Income 2000).

the list, until all tags have been inserted. It runs in $\mathrm{O}\left(n^{2}\right)$ time where $n$ is the number of tags. Another heuristic for the MLA problem is the PAIRWISE EXCHANGE MONTE CARLO (PWMC) method (Bhasker and Sahni, 1987): after applying NN, you repeatedly consider the exchange of two tags chosen at random, permuting them if it reduces the MLA cost. Another MONTE CARLO (MC) heuristic begins with the application of NN (Johnson et al., 2004): cut the list into two blocks at a random location, test if exchanging the two blocks reduces the MLA cost, if so proceed; repeat.

Additional display hints can be inserted in this list. For example, if two tags must absolutely be very close to each other, a GLUED token could be inserted. Also, if two tags can be permuted freely in the list, then a PERMUTABLE token could be inserted: the list could take the form of a PQ tree (Booth and Lueker, 1976).

\section{EXPERIMENTS}

Throughout these experiments, we used the Java version 1.6.0_02 from Sun Microsystems Inc. on an Apple MacPro machine with 2 Dual-Core Intel Xeon processors running at $2.66 \mathrm{GHz}$ and $2 \mathrm{GiB}$ of RAM.

\subsection{Iceberg-Based Computation}

To validate the generation of tag clouds from icebergs, we have run tests over the US Income 2000 data set (Hettich and Bay, 2000) (42 dimensions and about $2 \times 10^{5}$ facts) as well as a synthetic data set (18 dimensions and $2 \times 10^{4}$ facts) provided by Swivel (http://www.swivel.com/data_sets/ show/1002247). Figure 6 shows that while some tagcloud computations require several minutes, icebergbased computations can be much faster.
From each data set, we generated a 4-dimensional data cube. We used the COUNT function to aggregate data. Tag clouds were computed from each data cube using the iceberg approximation with different values of limit: the number of facts retained. We also implemented exact computations using temporary tables. We specified different values for tag-cloud size, limiting the maximum number of tags. For each iceberg limit value and tag-cloud size, we computed the entropy of the tag cloud, the false-positive and falsenegative indexes, and processing time for both of iceberg approximation and exact computation.

We plotted in Figure 7 the false-positive and falsenegative indexes as a function of the relative entropy (entropy/log (tag-cloud size $)$ ) using various iceberg limit values $(150,600,1200,4800$, and 19600) and various tag-cloud sizes (50, 100, 150, and 200), for a total of 20 tag clouds per dimension. The $\mathrm{Y}$ axis is in a logarithmic scale. Points having their indexes equal to zero are not displayed. As discussed in Section 4, false-positive and false-negative indexes should be low when the entropy is low. We verify that for low-entropy values $\left(<\frac{3}{4} \log (\right.$ tag-cloud size $)$ ), the indexes are always close to zero which indicates a good approximation. Meanwhile, small iceberg cuboids can be processed much faster.

\subsection{Similarity Computation}

Using our two data sets, we tested the NN, PWMC, and MC heuristics using both the cosine and the Tanimoto similarity measures. From data cubes made of all available dimensions, we used all possible 1tag clouds, using successively all other dimensions as clustering dimension for a total of $2 \times(18 \times 17+42 \times$ $41)=4056$ layout optimizations. The iceberg limit value was set at 150 . The MC heuristic never fared better than $\mathrm{NN}$, even when considering a very large number of random block permutations: we rejected this heuristic as ineffective. However, as Figure 8 shows, the PWMC heuristic can sometimes significantly outperform NN when a large number (1000) of tag exchanges are considered, but it only outperforms NN by more than $20 \%$ in less than $5 \%$ of all layout optimizations. Meanwhile, PWMC can be several order of magnitudes slower than NN: NN is 10 times faster than PWMC with 100 exchanges and 70 times faster than PWMC with 1000 exchanges. Computing the similarity function over an iceberg cuboid was moderately expensive $(0.07 \mathrm{~s})$ for a small iceberg cuboid (limit set to 150 cells): the exact computation of the similarity function can dwarf the cost of the heuristics (NN and PWMC) over a moderately large data set. Informal tests suggest that $\mathrm{NN}$ computed over a small 


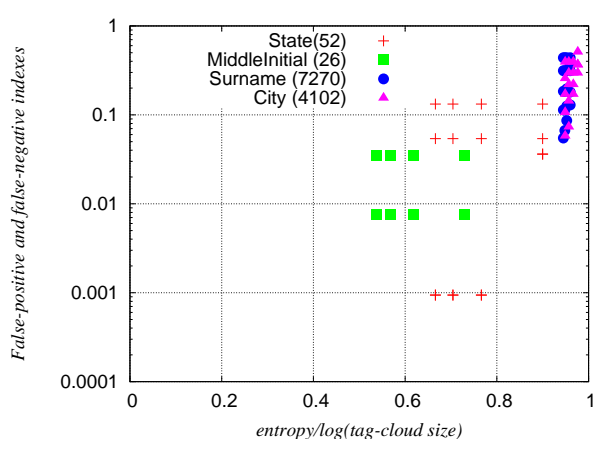

(a) Swivel

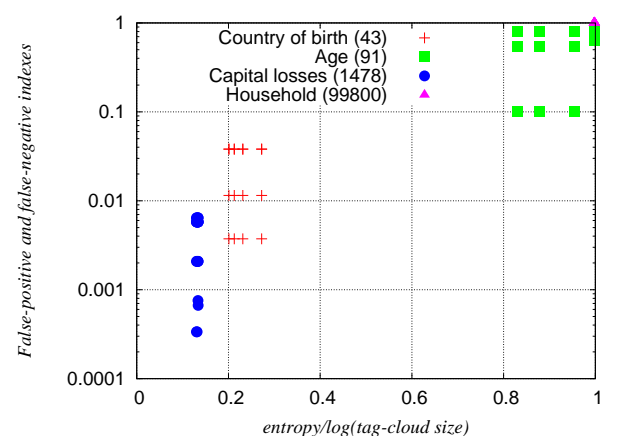

(b) US Income 2000

Figure 7: False-negative and false-positive indexes ( 0 is best, 1 is worst), values under 0.0001 are not included

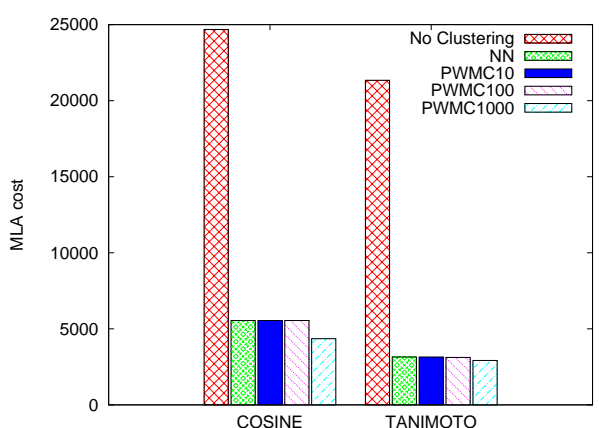

(a) Displaying dimension "Givenname" and clustering by "State" (Swivel)

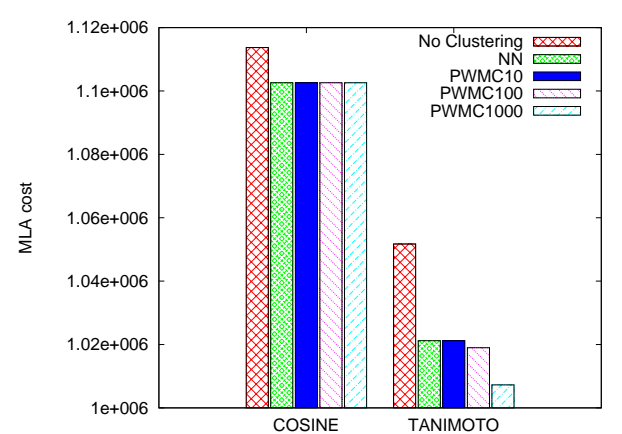

(b) Displaying dimension "HHDFMX" and clustering by "ARACE" (US Income 2000)

Figure 8: MLA costs for two examples: the PWMC heuristic was applied using 10, 100 and 1000 random exchanges.

iceberg cuboid provides significant visual layouts.

\section{CONCLUSION}

According to our experimental results, precomputing a single iceberg cuboid per data cube allows to generate adequate approximate tag clouds online. Combined with modern Web technologies such as AJAX and JSON, it provides a responsive application. However, we plan to make more precise the relationship between iceberg cubes, entropy, dimension sizes, and our quality indexes. Yet another approach to compute tag clouds quickly may be to use a bitmap index (O'Neil and Quass, 1997). While we built a Web 2.0 with support for numerous collaborations features such as permalinks, tag-cloud embeddings with iframe elements, we still need to experiment with live users. Our approach to multidimensional tag clouds has been to rely on $k$-tags. However, this approach might not be appropriate when a dimension has a linear flow such as time or latitude. A more appropriate approach is to allow the use of a slider (Russell, 2006) tying several tag clouds, each one corresponding to a given attribute value.

\section{ACKNOWLEDGMENTS}

The second author is supported by NSERC grant 261437 and FQRNT grant 112381. The third author is supported by NSERC grant OGP0009184 and FQRNT grant PR-119731. The authors wish to thank Owen Kaser from UNB for his contributions.

\section{REFERENCES}

Alon, N., Matias, Y., and Szegedy, M. (1996). The space complexity of approximating the frequency moments. In STOC '96, pages 20-29.

Ben Messaoud, R., Boussaid, O., and Loudcher Rabaséda, S. (2006). Efficient multidimensional data represen- 
tations based on multiple correspondence analysis. In $K D D^{\prime} 06$, pages 662-667.

Bhasker, J. and Sahni, S. (1987). Optimal linear arrangement of circuit components. J. VLSI Comp. Syst., 2(1):87-109.

Body, M., Miquel, M., Bédard, Y., and Tchounikine, A. (2002). A multidimensional and multiversion structure for OLAP applications. In DOLAP '02, pages $1-6$.

Booth, K. S. and Lueker, G. S. (1976). Testing for the consecutive ones property, interval graphs, and graph planarity using PQ-tree algorithms. Journal of Computer and System Sciences, 13:335-379.

Butler, D. (2007). Data sharing: the next generation. $\mathrm{Na}$ ture, 446(7131):1-10.

Carey, M. J. and Kossmann, D. (1997). On saying "enough already!” in SQL. In SIGMOD'97, pages 219-230.

Chazelle, B. (1988). A functional approach to data structures and its use in multidimensional searching. SIAM J. Comput., 17(3):427-462.

Chen, Q., Dayal, U., and Hsu, M. (2000). OLAP-based data mining for business intelligence applications in telecommunications and e-commerce. In DNIS 'OO, pages 1-19.

Chung, Y., Yang, W., and Kim, M. (2007). An efficient, robust method for processing of partial top-k/bottom-k queries using the RD-tree in OLAP. Decision Support Systems, 43(2):313-321.

Codd, E. (1993). Providing OLAP (on-line analytical processing) to user-analysis: an IT mandate. Technical report, E.F. Codd and Associates.

Cormode, G. and Muthukrishnan, S. (2005). What's hot and what's not: tracking most frequent items dynamically. ACM Trans. Database Syst., 30(1):249-278.

Donjerkovic, D. and Ramakrishnan, R. (1999). Probabilistic optimization of top n queries. In $V L D B^{\prime} 99$, pages 411-422.

Feige, U. and Lee, J. R. (2007). An improved approximation ratio for the minimum linear arrangement problem. Inf. Process. Lett., 101(1):26-29.

Gray, J., Bosworth, A., Layman, A., and Pirahesh, H. (1996). Data cube: A relational aggregation operator generalizing group-by, cross-tab, and sub-total. In ICDE' '96, pages 152-159.

Green, T. J., Karvounarakis, G., Taylor, N. E., Biton, O., Ives, Z. G., and Tannen, V. (2007). ORCHESTRA: facilitating collaborative data sharing. In SIGMOD '07, pages 1131-1133, New York, NY, USA. ACM.

Hassan-Montero, Y. and Herrero-Solana, V. (2006). Improving tag-clouds as visual information retrieval interfaces. In InSciT'06.

Havenstein, H. (2003). BI vendors seek to tap end-user power: New class of tools built to reap user knowledge for customizing analytic applications. InfoWorld, 22:20-21.

Heer, J., Viégas, F. B., and Wattenberg, M. (2007). Voyagers and voyeurs: supporting asynchronous collaborative information visualization. In $\mathrm{CHI}$ '07, pages 1029-1038.

Hettich, S. and Bay, S. D. (2000). The UCI KDD archive. http://kdd.ics.uci.edu [Online; accessed 21/12/2007]

IBM (2007). Many Eyes. http://services. alphaworks.ibm.com/manyeyes/ [Online; accessed 7-6-2007].
Jaffe, A., Naaman, M., Tassa, T., and Davis, M. (2006). Generating summaries and visualization for large collections of geo-referenced photographs. In MIR '06, pages 89-98.

Johnson, D., Krishnan, S., Chhugani, J., Kumar, S., and Venkatasubramanian, S. (2004). Compressing large boolean matrices using reordering techniques. In $V L D B^{\prime} 04$, pages 13-23.

Kaser, O. and Lemire, D. (2007). Tag-cloud drawing: Algorithms for cloud visualization. In WWW 2007 - Tagging and Metadata for Social Information Organization

Loh, Z., Ling, T., Ang, C., and Lee, S. (2002a). Adaptive method for range top-k queries in OLAP data cubes. In $D E X A^{\prime} 02$, pages 648-657.

Loh, Z. X., Ling, T. W., Ang, C. H., and Lee, S. Y. (2002b). Analysis of pre-computed partition top method for range top-k queries in OLAP data cubes. In CIKM'O2, pages $60-67$.

Luo, Z., Ling, T., Ang, C., Lee, S., and Cui, B. (2001). Range top/bottom $\mathrm{k}$ queries in OLAP sparse data cubes. In $D E X A^{\prime} 01$, pages 678-687.

Maniatis, A., Vassiliadis, P., Skiadopoulos, S., Vassiliou, Y., Mavrogonatos, G., and Michalarias, I. (2005). A presentation model \& non-traditional visualization for OLAP. International Journal of Data Warehousing and Mining, 1:1-36.

Millen, D. R., Feinberg, J., and Kerr, B. (2006). Dogear: Social bookmarking in the enterprise. In $\mathrm{CHI}$ 'O6, pages 111-120.

Morzy, T. and Wrembel, R. (2004). On querying versions of multiversion data warehouse. In DOLAP '04, pages 92-101

O'Neil, P. and Quass, D. (1997). Improved query performance with variant indexes. In SIGMOD' 97 , pages 38-49.

Poon, C. (2003). Dynamic orthogonal range queries in OLAP. Theoretical Computer Science, 296(3):487510

Rivadeneira, A. W., Gruen, D. M., Muller, M. J., and Millen, D. R. (2007). Getting our head in the clouds: toward evaluation studies of tagclouds. In $\mathrm{CHI}^{\prime} 07$, pages 995-998.

Russell, T. (2006). cloudalicious: folksonomy over time. In JCDL'06, pages 364-364.

Seokjin, H., Moon, B., and Sukho, L. (2005). Efficient execution of range top-k queries in aggregate r-trees. $I E$ ICE - Transactions on Information and Systems, E88$\mathrm{D}(11): 2544-2554$.

Swivel, Inc (2007). Swivel. http://www. Swivel.com [Online; accessed 7-6-2007].

Taylor, N. E. and Ives, Z. G. (2006). Reconciling while tolerating disagreement in collaborative data sharing. In SIGMOD '06, pages 13-24, New York, NY, USA. ACM.

Techapichetvanich, K. and Datta, A. (2005). Interactive visualization for OLAP. In ICCSA '05, pages 206-214.

Wattenberg, M. and Kriss, J. (2006). Designing for social data analysis. IEEE Transactions on Visualization and Computer Graphics, 12(4):549-557.

Wu, P., Sismanis, Y., and Reinwald, B. (2007). Towards keyword-driven analytical processing. In SIGMOD '07, pages 617-628. 